\title{
Interpretation of Rotation Curves of Giant Galaxies in the Local Group with the Truncated Disk Model: the Milky Way
}

\author{
I. I. Nikiforov and I. V. Petrovskaya ${ }^{1}$ \\ Sobolev Astronomical Institute, St. Petersburg University, \\ Bibliotechnaya pl. 2, 198904 St. Petersburg, Russia
}

S. Ninković

\section{Astronomical Observatory, Volgina 7, 11050 Belgrade, Yugoslavia}

\section{Introduction}

In some external edge-on spiral galaxies, such as NGC 891, 4244, 4565 and 5907 , the observed light distribution exhibits a sharp truncation in the outer parts (e.g., Casertano 1983, hereafter C83). As a rule, such galaxies are known to be warped in their gas disk, the deformation starting at about their optical cut-off radius. These facts suggest a truncation in the disk density distribution. Moreover, the rotation velocity of these galaxies starts decreasing at about the same radius, which can also be considered as the "signature" of truncation (see C83).

Both giant spiral galaxies in the Local Group, the Milky Way and M31, have a dip in the average rotation velocity which can be produced by a truncation of the galactic disk. Ninković \& Petrovskaya (1992) constructed from Haud's (1981) rotation curve for M31 a model including, like C83's for NGC 5907, a truncated exponential disk and a halo. The truncation distance was found to be close to the radius beyond which warping of the hydrogen layer of M31 begins.

For our Galaxy a preliminary mass distribution model was constructed which reproduces a dip in rotation velocity at about the solar radius $R_{0}$ by Petrovskaya (1992). In this paper we present results of a more comprehensive study for the Galactic rotation curve constructed in Nikiforov (1999) (hereafter N99) with $R_{0}=8.2 \mathrm{kpc}$ and the solar circular velocity of $216.5 \mathrm{~km} \mathrm{~s}^{-1}$.

\section{Rotation curve of the Milky Way}

We use the recent data on the rotation of the neutral hydrogen from the whole 21$\mathrm{cm}$ line profile and the tangent points, and of molecular clouds with $\mathrm{CO}$ emission (for details see N99). Our rotation curve of the Milky Way (Figure 1 in N99 and Figure 1 in this paper) has a significant dip, on average, of $\sim 20 \mathrm{~km} \mathrm{~s}^{-1}$ between relative radii of $x \equiv R / R_{0}=0.75$ and 1.15. At larger distances the rotation velocity $\theta$ initially increases and then remains virtually constant at $x>1.7-1.8$.

${ }^{1}$ Deceased on August 11th, 1999 


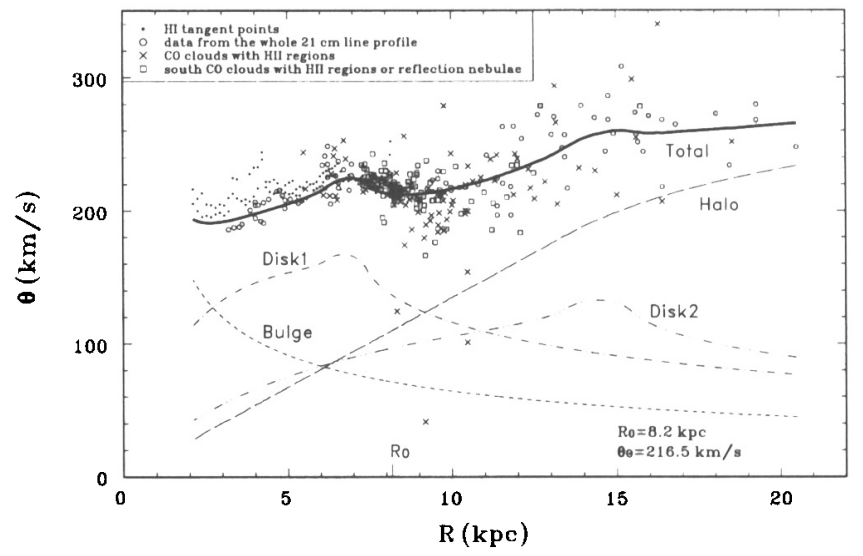

Figure 1. A mass model of the Milky Way fitted to the rotation curve from $\mathrm{HI}$ and $\mathrm{CO}$ data.

It is usual practice to ignore these details of the rotation curve in constructing a mass distribution model for our Galaxy (e.g., Merrifield 1992). On the other hand, Lépine \& Amaral's (1995) attempt to reproduce the minimum at $x \sim 1.1$ exclusively by adding a perturbation from spiral arms fared poorly. Besides, it is well known that the Milky Way also has a warp of the $\mathrm{H}$ I layer for $x>1.1$ (e.g., Gerasimov \& Petrovskaya 1990), the thickness of the layer starting to rise steeply at $x>0.8-0.9$. On this basis we suppose that the dip in density of the Galactic disk at $R<R_{0}$ could, at least in part, explain the decrease in $\theta$ near $R_{0}$.

\section{3. $\quad$ Model}

We constructed a four-component model for the Milky Way consisting of two truncated exponential disks (inner and extended), a bulge and a "dark halo". The inner disk is required to reproduce the $\operatorname{dip}$ in $\theta$ at $x<1$ by a fall in disk density. The extended disk is called for a representation of the disk matter in the vicinity of the Sun and in the outer Galaxy where the disk component has a cut-off at $x \sim 1.7$ (Porcel et al. 1997 and references therein). The "break" in the rotation curve at $x=1.7-1.8$ (Figure 1) may be associated with the latter detail.

We use C83's expressions for density distribution in the "softly truncated disk":

$$
\begin{gathered}
\rho(R, z)=\rho_{0}(R)\left[\operatorname{ch}\left(z / z_{0}\right)\right]^{-2} \\
\rho_{0}(R)= \begin{cases}\rho_{00} \exp (-R / h), & R \leq R_{\mathrm{d}} \\
\rho_{0}\left(R_{\mathrm{d}}\right)\left[1-\left(R-R_{\mathrm{d}}\right) / \delta\right], & R_{\mathrm{d}} \leq R \leq R_{\mathrm{d}}+\delta \\
0, & R \geq R_{\mathrm{d}}+\delta,\end{cases}
\end{gathered}
$$


where $R$ is the distance from the Galactic axis, $z$ is the distance from the Galactic plane, $z_{0}$ is the disk half-thickness $(=100 \mathrm{pc}$, i.e. the mean thickness of $\mathrm{H} \mathrm{I}$ and CO layers), $R_{\mathrm{d}}$ is the cut-off radius, $\delta$ is the cut-off length. The central surface density $\mu_{0}$ equals $2 \rho_{00} z_{0}$.

Note that we do not suggest any physical difference between the two disks; this formal decomposition is taken only to represent dips in disk density.

As the bulge model, we use a truncated Hubble law spheroid:

$$
\begin{gathered}
\rho(q)= \begin{cases}\rho(0)\left[1+\left(q / q_{\mathrm{b}}\right)^{2}\right]^{-3 / 2}, & q \leq a_{\mathrm{b}} \\
0, & q>a_{\mathrm{b}},\end{cases} \\
q=\left[R^{2}+(z / \varepsilon)^{2}\right]^{1 / 2}, \quad \varepsilon=0.6 .
\end{gathered}
$$

This component has only marginal dynamical significance in the range of distances probed by our rotation curve.

The density distribution used for the halo is constant in the inner part and pseudo-isothermal in the outer part:

$$
\begin{gathered}
\rho(q)= \begin{cases}A\left[\frac{1}{1+\left(q / q_{\mathrm{h}}\right)^{2}}-\frac{1}{1+\left(a_{\mathrm{h}} / q_{\mathrm{h}}\right)^{2}}\right], & R_{\mathrm{h}} \leq q \leq a_{\mathrm{h}} \\
\rho\left(R_{\mathrm{h}}\right), & q \leq R_{\mathrm{h}} \\
0, \quad & q \geq a_{\mathrm{h}},\end{cases} \\
\quad q=\left(R^{2}+z^{2}\right)^{1 / 2} .
\end{gathered}
$$

This complication is based on our modelling results: we found the scale parameter $q_{\mathrm{h}}$ to increase without limit during the fitting procedure for the pure pseudo-isothermal model.

\section{Model Fitting and Results}

By assuming values of model parameters $\left(q_{\mathrm{b}}, q_{\mathrm{h}}\right.$ and $\left.a_{\mathrm{h}}\right)$, which cannot be found uniquely, we derived others by a fitting to the rotation data. The best-fit solution, which minimizes the quantity $\sum_{j}\left(\theta_{\text {obs }}-\theta_{\text {mod }}\right)_{j}^{2} / \sigma_{\theta_{j}}$, was found by quasiNewton algorithm for finding minimum of a function of more then one variables. The mass of the extended disk was normalized to give a local surface density of $50 M_{\odot} \mathrm{pc}^{-2}$ (Kuijken \& Gilmore 1989, Holmberg \& Flynn 2000). Results are given in Table 1. The mass of the Galactic disk is $5.85 \times 10^{10} M_{\odot}$, the total mass of the Galaxy in the central $20.5 \mathrm{kpc}$ of this model is $(3.3 \pm 0.4) \times 10^{11} M_{\odot}$.

The mass model is presented in Figure 1. This model reproduces the dip in $\theta$ of $13 \mathrm{~km} \mathrm{~s}^{-1}$ at $R \sim R_{0}$ and the "break" at $x=1.7-1.8$. The model dip is in good agreement with the observed one from the $\mathrm{HI}\left(\approx 10 \mathrm{~km} \mathrm{~s}^{-1}\right)$. The observed greater dip of $30 \mathrm{~km} \mathrm{~s}^{-1}$ from molecular clouds is most probably induced not by a drop in the disk density but by a perturbation from a spiral wave.

\section{Conclusion}

Rotation curves of both giant spiral galaxies in the Local Group can be reproduced in detail with the truncated exponential disk model. In the case of the 
Table 1. Mass model solution for the Milky Way galaxy.

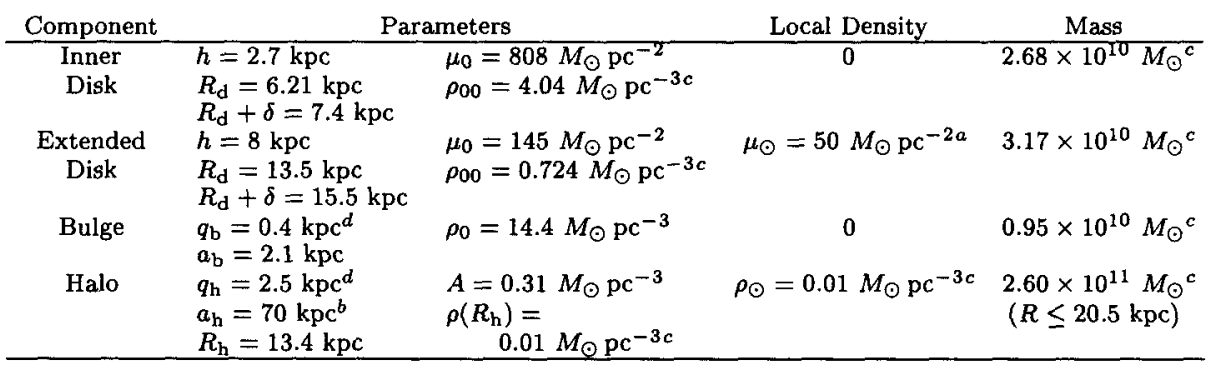

${ }^{a}$ Kuijken \& Gilmore (1989), Holmberg \& Flynn (2000)

${ }^{b}$ Ninković (1992)

${ }^{c}$ Calculated from found and accepted parameters

${ }^{d}$ Accepted after a tentative fitting

Milky Way, there is evidence for two density dips in the disk which leave their "signatures" on the rotation curve. Conceivably the generation of these truncations might be induced by interactions of giant galaxies with small satellites or by rare encounters with more massive but presently remote LG member. This may be considered as a special problem of the LG dynamics, the solution of which requires data on proper motions.

Acknowledgments. This work is partly supported by the Russian Foundation for Basic Research (grant no. 96-02-19636).

\section{References}

Casertano, S. 1983, MNRAS, 203, 735

Gerasimov, A. G., \& Petrovskaya, I. V. 1990, Kinematika i Fizika Nebesnykh Tel, 8, 17

Haud, U. 1984, Astrophys. and Space Sci., 104, 337

Holmberg, J., \& Flynn, C. 2000, MNRAS, in press

Kuijken, K., \& Gilmore, G. 1989, MNRAS, 239, 605

Lépine, J. R. D., \& Amaral, L. H. 1995, Mem. Soc. Astron. Ital., 66, 649

Merrifield, M. R. 1992, AJ, 103, 1552

Nikiforov, I. I. 2000, these proceedigns

Ninković, S. 1992, Astron. Nachr., 313, 83

Ninkivić, S., \& Petrovskaya, I. V. 1992, Sov. Astron., 36, 478

Petrovskaya, I. V. 1992, A\&A Trans., 3, 87

Porcel, C., Battaner, E., \& Jiménez-Vicente, J. 1997, A\&A, 322, 103 